\title{
Caring for children with disabilities in a foreign land: Experiences and perceptions of a group of Korean parents and professionals
}

\author{
Clara Choi ${ }^{1}$, Hong-Jae Park² and Mike O'Brien ${ }^{1}$
}

\begin{abstract}
INTRODUCTION: This article aims to provide an overview of the experiences of Korean immigrant parents raising children with disabilities in Aotearoa New Zealand, primarily focusing on their experience of disability services and systems.
\end{abstract}

METHODS: As the present exploratory study aimed to gain a deeper understanding of immigrant parents' experiences and perceptions, a qualitative approach was employed to collect rich and lived information from participants. Ten participants were recruited and interviewed among Korean parents of children with disabilities and professionals working with those families.

FINDINGS: Lack of trust among Korean parents living in Aotearoa New Zealand was frequently discussed by the participants in the present study. Lack of information around available services and alternative support and cultural barriers were often identified to have a significant impact on Korean parents' experiences with services. A sense of obligation to integrate into the host society and the services provided was also evident. Further, there was a contradictory perception between parents and associated professionals in relation to services' expectations of Korean parents.

CONCLUSION: From analysis of the findings, three main themes emerged: experiences of services and its relations with trust, cultural values and expectations, and looking to the future. The study suggests that there is a need for professionals, service providers and government to consider ways to build trusting relationships with Korean parents and their children with disabilities, and makes a number of recommendations.

KEYWORDS: children; disabilities; culture; parents of children with disabilities; migrant parents; Korea

\section{Introduction}

Caring for children with disabilities can be particularly challenging for immigrant parents. It is highly likely that migrants experience difficulties around the migration and resettlement processes (Cho, Singer, \& Brenner, 2000; Welterlin \& LeRue, 2007; Wong et al., 2004). Language barriers, along with cultural differences, can cause a range of challenges; in particular, raising children with disabilities can be extremely difficult in the host society. It is to be expected then that Korean immigrant parents of children with disabilities may face dual challenges - as immigrants, and also as parents of a child with disabilities.

The aim of this article is to explore the experiences of Korean immigrant parents raising children with disabilities in Aotearoa New Zealand. The article provides a critical overview of a range of other research and findings around the topic, including
${ }^{1}$ University of Auckland, New Zealand

${ }^{2}$ Western Sydney University, Australia

AOTEAROA

NEW ZEALAND SOCIAL WORK 29(4), 61-73.

CORRESPONDENCE TO: Clara Choi jcho199@aucklanduni.ac.nz 
general literature on disability, the Korean community and culture and cultural issues in relation to disability. There is a significant wealth of literature on more general experiences of, and issues with, raising a child with a disability but detailed discussion of this is beyond the scope of what is possible here (see Khanlou, Haque, Sheehan, \& Jones, 2015; Park \& Chung, 2014; Shin, 2002; Welterlin \& LeRue, 2007). Methodological and ethical considerations impacting on the present, small-scale exploratory research are discussed briefly. This article then explores the findings of the study around parents' experiences with services, as well as their expectations of the services provided. The article intends to provide human service professionals with research evidence that is specific to the Aotearoa New Zealand context to guide practice with immigrant parents of children with disabilities.

Though learning and gaining access to services can be difficult for any parents raising children with disabilities, those who are not members of the majority culture are likely to find these processes especially challenging. Studies reveal that families of the host society both anticipate and experience fewer barriers in gaining access to health services than families of other ethnic groups (Bailey, Skinner, Rodriguez, Gut, \& Correa, 1999). Furthermore, as evident in these studies (Bywaters, Ali, Fazil, \& Wallace, 2003; Fatimilehin \& Nadirshaw, 1994), families of an ethnic minority caring for children with disabilities frequently experience immediate or additional barriers to equality of opportunity and equality of treatment when compared with those families from the mainstream population.

Alongside these cultural and language issues, carers generally experience a tremendous amount of emotional distress while raising their children with disabilities (Fatimilehin \& Nadirshaw, 1994; Vickers, 2006; Wong et al., 2004). Though there are various ways different families and carers react to a child with a disability, they commonly express denial, shock and anger, which is all part of grief and loss processes. Feelings of powerlessness, guilt, despair, frustration, depression, fear, self-blame and confusion are also experienced (Fatimilehin \& Nadirshaw, 1994; Vickers, 2006). Yet, there are also parents who highlight the experiences of raising children with disabilities in a favourable light. As evident in studies (Cho et al., 2000; Park \& Chung, 2014), experiences of raising children with disabilities have been acknowledged to have provided opportunities for parents' personal growth as they gained positively from a challenging situation, and the experience offered them the opportunity to reflect on the bigger picture of life and expanded their perspective on life.

Alongside these general considerations, it is important to pay close attention to the significance of the impact of Korean cultural values on parenting and supporting children with disabilities. It is argued that, although traditional Korean values are influenced by many different factors, the most dominant one is clearly Confucianism (Hyun, 2001; Park \& Cho, 1995). It is believed that Confucianism has been powerful in shaping the behavioural pattern and structure of the family and communities (Park \& Cho, 1995). As Hyun (2001) noted:

To ensure harmony and order in the family and in society, one must abide by filial piety as the cardinal value... The Confucian code of conduct also prescribes the principles for the major interpersonal relations, including those between: ruler and minister, parent and child, older and younger brothers, and husband and wife. Wisdom, responsibility, and benevolence descend from the former [superior], and obedience, loyalty, and respect are expected of the latter. (pp. 205-206)

From research investigating Chinese cultural influences (largely impacted by Confucianism) on parental care-giving obligations toward children with disabilities 
(Holroyd, 2003), it was concluded that parents of children with disabilities go beyond the demands of mere duty. The study argued that, in such circumstances, compared to the obligations of parents with children who are living without impairment, parents with children with disabilities may voluntarily give of themselves in ways for which few cultural guidelines or expectations exist (Holroyd, 2003).

Further, looking specifically at Korean culture and cultural issues in relation to disabilities suggests that Korean people have a strong sense of collective group identity, and thus may experience and perceive raising children with disabilities differently from a more individualistic society, and may also hold different sets of expectations (Kim, Atkinson \& Yang, 1999; Kim-Rupnow, 2005). In contrast to a Western cultural ideology where uniqueness, autonomy and independence based on internal attributes (such as thoughts and emotions) serve as the primary guide for behaviour and cognition, the predominant cultural ideology in Korea is interpersonal connectedness and social conformity (Kim-Rupnow, 2005). It is argued that such social conformity, stressed in collectivist cultures, may result in greater social stigma. It is suggested that the social stigma of having disabilities will likely widen to other in-group members than in more individualistic cultures (KimRupnow, 2005). Furthermore, according to Kim-Rupnow's (2005) study, the majority of Korean people tend to avoid people with disabilities, often due to feelings of uneasiness and not knowing what to do. Thus, Korean people with disabilities are highly likely to experience isolation.

However, despite the concerns raised and issues evident regarding ethnic minority families caring for children with disabilities across the globe, there is a lack of studies undertaken around this issue in Aotearoa New Zealand. In particular, despite the fact that the Korean migrant population is one of the largest Asian groups in Aotearoa
New Zealand (Statistics New Zealand, 2013), little is known about their lives. Working with migrant children with disabilities requires an understanding of their cultural values, social needs and personal perceptions. Specifically, an emphasis on cultural diversity in the social work profession is likely to have a positive influence on both clients and professionals (Lindsay, Terault, Desmaris, King, \& Pierart, 2014), and contribute to a fully inclusive society for every New Zealander.

\section{Methodology}

As the study aims to gain a deeper understanding of immigrant parents' experiences and perceptions, a qualitative approach was employed to collect rich and lived information from participants. Questions were asked around the experiences of raising children with disabilities in Aotearoa New Zealand, their perceptions of the children's disabilities and special needs in this context, and their expectations regarding social support and services for their children.

Ten participants were recruited among Korean parents of children with disabilities and Korean professionals working with them. Using purposive sampling, seven parent participants were recruited who were identified to have the experience to answer the above questions, and three professional participants working in the disability sector were recruited to provide a more systematic overview and more objective, professional views on experiences and perceptions of Korean parents.

This small-scale exploratory study was conducted by an insider researcher which made it possible for the development of empathy towards participants. Within the migrant community, this had a positive influence on building rapport and relationship with participants. The first author is a Korean student with physical disabilities, and this insider position, as a person with disabilities and a member of the migrant community, made it possible 
for the participants to be more open and willing to participate in the study. Though the participants and the researchers held different beliefs, to a certain degree all the parties were able to build trust, share, understand and make connections with each other. All these steps helped to ensure the trustworthiness of the data was enhanced.

The study was approved by the University of Auckland Human Participants Ethics Committee.

\section{Sampling}

The participants in this study were recruited among Korean parents of children with disabilities and the professionals working with them. Professionals in the present study were individuals who belonged to a service provider or to an organisation providing disability services and which is directly funded by the government to provide support to people with disabilities. There were 10 participants in this study. Purposive sampling, using a snowballing approach, was applied to recruit participants in the Korean community. There were two groups, seven parents (five mothers and two fathers) and three professionals (two females and one male). Participants who fell under both sample categories were asked if they had a preference as to how they would like to be identified; if not these participants were classified according to statements they made during the interview (that is, if the statements they made during the interview were predominantly from a professional viewpoint they were considered as professionals, and if the statements were from a parenting viewpoint they were considered as parents). Participants were from a variety of backgrounds and profiles: participants' ages ranged from early 20 s to late $60 \mathrm{~s}$; the levels of education ranged from secondary education to tertiary and above, and years of residence in Aotearoa New Zealand ranged from less than five to more than 21 .

\section{Data collection}

Data were collected through individual interviews exploring their experiences and perceptions around raising children with impairments in Aotearoa New Zealand, their main formal and informal support, and expectations around care provisions. Both professionals and parents were asked similar questions and, as mentioned previously, professionals were recruited to provide professional views around the experiences and perceptions of Korean parents in Aotearoa New Zealand. Interviews were semistructured, using an interview guide. Each interview lasted approximately 60 minutes, and data transcription resulted in approximately 15 pages for each participant. Interviews were conducted in the participants' native language (Korean) although they could also use the English language if they wanted. Interviews were recorded using a digital voice recorder.

\section{Data analysis}

The data collected from interviews were analysed through a conventional analysis method where coding categories were derived directly from the text data (Hsieh \& Shannon, 2005). All authors participated in this process to verify and increase the validity of the emergent themes. From multiple readings of the transcripts of all interviews, it emerged that there were a number of subjects that were frequently visited by participants. Those subjects became the initial codes. After a process of coding and recoding, two major themes emerged: experiences with services, and expectations of services. Under each theme a number of sub-themes were identified.

\section{Methodological limitations}

The overall limitations of qualitative research methods were unavoidable in the present study. As the study had limitations around a sample size of 10 , 
the present study lacks the power to be generalised to a wider population (Monette, Sullivan, \& DeJong, 2008). However, this did not detract from the main objective of this study: to obtain rich and in-depth data on how two groups of participants perceive and experience services. The exploratory nature of the research meant that this rich data both informed this project and provides a base for further study in this area. Furthermore, participants were recruited only from Auckland (an urban setting). The experiences, perceptions and expectations of parents outside Auckland were not included in this present study; such inclusion might have resulted in different findings and discussion. Therefore, the present study provides a basic understanding about the experiences of a group of Korean migrant parents raising a child with disabilities in Auckland.

\section{Findings}

\section{Experiences with the services}

Lack of trust among Korean parents living in Aotearoa New Zealand was frequently discussed by the participants in the present study. Parents expressed anxiety over whether their children were receiving appropriate support and service. For example, a mother whose son was currently living in a group home constantly expressed her concern about whether her son was treated with respect. She was especially concerned about whether his rights were being met when she was not around.

Things that I am worried about is the things they may do when they are not seen by others, ... I do understand that within relationships, even among people without disabilities, it is realistically very difficult to ensure all your rights, but if you have disabilities, this gets really hard unless someone is not watching over you ... My son stays in a group home, and as a mother, I get the feeling that these things are not happening in [the] right manner. (Cho)
While communicating a lack of trust towards the services and professionals, parent participants also articulated the need for closer monitoring of service providers' performance. For instance, another mother shared how she felt her son's basic needs and rights were not being met by services; she expressed what she felt was a need for closer performance monitoring. She commented that such professional performance has led to reduced trust towards the services offered.

It was also evident that such a lack of trust or anxiety about the services may create a barrier to service use. For instance, a father of a daughter with autism disclosed that, when he felt that a teacher from a specialised school could not meet the expectations of his family, and that she was not competent enough to carry out her role as a professional, he considered withdrawing his daughter from the service and home-schooling her.

Emotional challenges were not only experienced at family and individual levels, but were also experienced while engaging with services. There were some cases where parents felt emotionally challenged by service providers. As an example, one mother recalled her memory of feeling shame and humiliation when service providers came to assess her family at her place. She recalled:

I was living in an environment where, as a human and as a housewife, I didn't want to show anyone my situation ... I felt shame and to certain degree insulted. (Cho)

Korean parents raising children with disabilities and the professionals working with them who participated in this study repeatedly raised their concerns around their lack of knowledge about available services and also having limited understanding around how the general health care system operates in Aotearoa New Zealand. 
A number of parents reported experiencing difficulties finding and engaging with services. For instance, a mother of a son with autism experienced difficulties letting people know her situation so that she could get support. She explained, because she previously lived in Korea where the welfare system is rather different, she did not understand what was expected of her in order to receive support in Aotearoa New Zealand.

The language barrier was identified as one of the major contributing factors to these issues by both professional and parent participant groups. Limited English proficiency impacted on parent participants seeking information, engaging with support services and communicating their desires and needs freely. A professional who is also a parent of a child with disabilities noted:

It was more like problems that you face as a migrant, not only because I have a child with disabilities. I didn't know where to look for information, what kind of supports were available, and how to communicate with teachers, I really had no idea. (Kim)

Participants from both groups frequently discussed how, as Korean parents, they feel an obligation to integrate into the host society even if they are somewhat dissatisfied with the services provided. As a member of a minority group, they felt that there are not many services that focus on, and fit with, Korean culture, thus they feel the need to adapt to existing services. A professional participant approached the matter from a cultural perspective. He noted:

They tend to think very passive ... they wonder if they are entitled to such service ... I think it's because they, Korean parents, are not used to social service or welfare services ... Korean parents find the concept "partnership" very difficult, as there was no such concept for them and they were always a passive recipient. (Lee)
A professional noted that when children go into a residential house or group home and live independently from their parents, there are difficulties due to cultural differences. She often feels that Korean parents are not satisfied with the services provided. However, according to her experiences, Korean parents generally feel that their children need to adjust to Aotearoa New Zealand culture as they have to receive services and live under the health care system that was developed to serve New Zealanders.

Further, Korean parents in the study found their experience of waiting for services in Aotearoa New Zealand challenging. For instance, a participant who was a specialist teacher in Korea, and had been working with children with disabilities within Aotearoa New Zealand for a number of years stated:

I get the feeling that the general process of everything is very slow. So when parents are waiting for the process, there is a gap ... So between that gaps sometime parents have to financially support the child privately ... because the process is way too slow. (Jung)

This has caused nervousness and increased anxiety among Korean parents raising children with disabilities in Aotearoa New Zealand as, in Korea, the fundamental bases for good services are accuracy and speed.

Throughout the interviews, it was evident that parent participants have limited or no support from other family members or friends. It was perceived that being migrants has set some limitations on having personal and informal support. They noted that, because their children are different from children who do not have disabilities; it is difficult to be supported by people from their personal networks. A father of a child with autism stated:

We don't know other families here. And even if we know a lot of people, because my daughter has disabilities, the way we think is different from normal people. (Kwak) 
Some participants had different perceptions about parenting from how the current disability services are operating. Participants in this study expressed their anxiety around current services and felt that they are not maximising the opportunities for their children to reach their full potential, but are rather overly focused on avoiding or minimising potential risks in the process of service provision. They see a need for their children to experience more and to push the boundaries.

I go to different places with the children I work with. This holiday, we are going to a ski camp. In some way this is very risky thing to do ... But I believe that, when they experience this kind of thing, their quality of life changes. I think that's the difference, difference between being educated and not. (Jung)

Further, it was evident in the present study that there is a relationship between the risk-focused practice and a decline in confidence and trust in professionals. For instance, both parent and professional participants have complained about the amount of auditing and paperwork, which were perceived to be carried out to avoid any potential blame for their work, creating barriers and limitations for professionals to provide services and opportunities for their children to reach their full potential. It was perceived that trust in professionals had been substituted by audit and excessive amount of paperwork.

In contrast to the difficulties discussed earlier, many participants also acknowledged positive aspects of their experiences in raising children with disabilities in Aotearoa New Zealand. Participants often perceived Aotearoa New Zealand disability services as very effective and supportive; they were also very thankful and satisfied with the general societal view around people with disabilities in New Zealand. Some participants made comparisons between raising children with disabilities in Korea and Aotearoa New Zealand.
I get the feeling, in terms of culture, New Zealand is much better. And I heard that this is why many parents of children with disabilities migrate to New Zealand. Because of the inhumane treatment they get in Korea .... and I think parents generally accept that perception around children with disabilities here in

New Zealand is better than Korea, and that children are happy. (Jung)

\section{Expectations}

From the interviews, there emerged a contradictory perception between parents and professionals working with them in relation to the expectations of Korean parents to services. The data revealed that professionals felt that parents had high expectations of services.

It's really hard to say what their expectations are ... but I can say that what parents want, their expectations are never ending ... and because it's never ending, there is no service that can meet their expectation. (Park)

However, parents interviewed had a contrary view stating they do not expect too much from services, especially from services provided from the Korean community. This was often associated with having low expectations of volunteers. Parent participants placed more emphasis on their children having somewhere to go and to spend time.

To be honest, I don't expect too much from services. The important thing for me and my family is that my son has a place to go to during weekends. I don't expect that the service and program[me]s provided within [the] Korean community can improve my son. Because they are volunteers, there is a real limit to it, they don't hold responsibility. And they don't have specialized skills or anything. (Kim)

Parents' expectations of their children with disabilities around the use of language were 
also frequently discussed by both parent and professional participants in this study. These participants shared certain expectations.

A number of participants revealed that parents experience difficulties around deciding which language their children should use. This is an issue for Korean parents as children need to communicate with family members in Korean at home, but when they are in school or other services they need to use English to communicate. A professional noted:

From [the] parents' view, children need to speak Korean at home, and at schools they need to speak English ... they know that they are going to be cared for and supported within Kiwi society and study within an environment where English is the first language. So for Korean parents, they are concerned about how to balance this issue around language. (Park)

She also expressed that there are issues that are sometimes raised due to miscommunication between children with disabilities and professionals. She noted that professionals are always cautious as some children may have difficulties communicating their needs or expressing themselves when in dangerous or unsafe situations.

The data revealed that most participants were concerned about the disabled person's future; this is especially relevant in relation to after their parents' passing. Many were anxious that they themselves or other disabled persons' parents are not prepared for their children's future care and that children are not ready for independent living without parental care. One professional participant expressed his concerns around parents being so preoccupied with caring for children that there is no room for preparation for the future to take place. He argued that education and training for independence must be taken into consideration when raising a child with disabilities.

On the other hand, some participants had gone beyond expectations and prepared some plans for their children's future. There were a number of different ideas around children's future care plans discussed throughout the interviews that were considered by both parents and professionals. These plans ranged from very detailed, day-to-day plans to comprehensive organisational-level preparations. One example of an organisational-level future care plan is the "Cookie Project." This is a project initiated by a Korean service provider in the Auckland community to support and create job opportunities for Korean children with disabilities. They teach children how to bake and help them make sales to the local churches. The ultimate goal of the project is to make enough profit through these activities to set up an organisation that is established and operated by Korean children with disabilities to lead more independent lives within the Korean disability community.

Furthermore, there were a number of participants who saw a need for more Korean professionals to be involved in the field. A professional participant further commented that, as Korean people are starting to know of other parents with children with disabilities within the community, perhaps they could think about establishing an organisation for Korean children with disabilities and provide support and services for them. He went on to explain how such involvement can create employment opportunities for Korean parents, and that this might lead to greater financial independence for Korean parents with children with disabilities as well as for the children themselves.

\section{Discussion}

\section{Experience of services and its relationship with trust}

The present study revealed significant issues in relation to Korean parents' experiences using disability services and an associated lack of trust. This mistrust towards professionals and service providers was 
evident in the present study in many areas. Feelings of anxiousness around lacking knowledge about available services and having limited understanding about how the general health care system operates were perceived to be significant issues for Korean parents caring for children with disabilities.

The findings highlight the need for service providers to work at building trust with Korean parents. This could be achieved by starting from a place of genuinely listening to and understanding the values and expectation of the families (King, Desmarais, Lindsay, Piérart, \& Tétreault, 2015).

Trust would also be enhanced by making information more accessible. Training and educating volunteers in the Korean community working with children with disability might be valuable in increasing trust in services among Korean parents. From the findings of the study it appears that increasing public awareness around disabilities may enable people and family members to consider disability as more of a public issue rather than just as a personal problem. This might enable Korean parents to feel less reluctant to go to public places and enable society to be more tolerant and accepting; this might lead to more opportunities for Korean children with disabilities to explore and experience a wider range of activities.

Emotional distress was revealed to be a significant issue for Korean migrant parents raising children with disabilities. Yet, it was evident that Korean parents face various challenges and additional emotional distress as a result of both frequenting public places and engaging with service providers. This often led to a loss of faith in professionals and a questioning of the quality of professionals working in the field.

The study findings recorded a discrepancy between Korean parents and professionals regarding their perceptions around expectations of parents towards support services. Professionals experienced that parents had high expectations of what services could and should provide, whereas parents had rather low expectations of services. This is an area that needs further research to discover the reasons behind this perceived discrepancy. Both Korean parents and professional participants were concerned that the system in Aotearoa New Zealand is not well structured and focuses too much on risks. Parents were generally anxious that such risk-focused services, where risk has become the key aspect of the services, might decrease opportunities for children with disabilities to reach their full potential. They seemed to fear that current Aotearoa New Zealand services may not assist their children to meet their full potential.

Despite the reservations and poor experiences, some Korean parent participants acknowledged having experienced positive aspects while raising their children with disabilities in this country. It was acknowledged that the societal perception and beliefs around people with disabilities within Aotearoa New Zealand are much more positive than they are in Korea. Raising a child with disability is believed to have offered Korean parents raising children with disabilities the opportunity to view life through a different lens. As is evident, there is a tension between being disappointed with the services and being pleased. However, it is not clear why such contrasts exist; thus, this is an area for further work.

\section{Cultural values and expectations}

Another significant issue raised in the present study was cultural values and their impact on expectations of Korean parents caring for children with disabilities in Aotearoa New Zealand. The Korean parents interviewed had a very strong sense of responsibility and obligation in terms of caring for their child; this is believed to be influenced by Confucianism (Hyun, 2001; Lee, 1983; Park \& Cho, 1995). From the analysis of the findings, a potential relationship between Confucian principles around responsibility and obligation, and 
trust is evident. It is likely that parents' expectations of services are influenced by Confucian principles that come with their carer roles and, when they perceive that services are not meeting these sets of responsibilities and obligations, they find it difficult to trust services and hence are reluctant to share their duty of caring for their children. This might also explain why professionals feel these parents have high expectations. Engaging with social services and professionals is further complicated for these parents due to their migrant status. As evident in the findings, they experienced power differences and often perceived themselves as passive recipients who have to simply adjust and conform to the services and society.

Studies (Cho et al., 2000; Hyun, 2001; Park \& Cho, 1995) on the impact of informal support on managing children with disabilities among Asian families suggest that such support is one of the most significant and beneficial supports within Asian families with disabilities. However, it was evident in the present study that Korean parents raising children with disabilities in Aotearoa New Zealand often have no informal support; they also experience a lack of interpersonal relationships and lack a sense of strong relationship within the Korean New Zealand community. The Korean parents in this study tended not to be very open about their child's impairment with people outside of their family due to negative messages from those in their informal networks. Further, as discussed earlier, the emphasis on social conformity embedded within collectivist cultures may result in greater social stigma. Accordingly, the fear of confidentiality being breached and families being exposed to social stigma may have an impact on Korean parents' help-seeking behaviour in a relatively small Korean community. Further, their status as migrants is perceived to be one of the factors impacting on such a lack of informal support, but such experiences were linked with a lack of confidence as to whether their children with disabilities would be able to receive appropriate and adequate care through informal support. It was generally believed that other family members and friends are unlikely to understand the experiences and needs of children with disabilities. In other words, there was a high level of expectations of care providers while there was a rather low level of trust. Such perceived high expectations around care provision based on parents' high level of responsibility and obligation results in a lack of trust in others and in becoming more hesitant to share their caring duties with people who do not share their values.

The findings of the present study articulated the need for professionals to support and assist Korean parents in organising or taking part in self-help or peer-support groups to support each other. For professionals supporting children with disabilities and their families, it may be valuable to offer children and families the opportunity to meet children or families who are in a similar situation. However, since Korean parents in Aotearoa New Zealand appeared to have comparatively fewer parental networks and presented as more exclusive and passive due to the reasons discussed earlier, there is a need for professionals to approach Korean parents (while acknowledging their differences in culture). This can be achieved through having an understanding around general Korean customs and culture, the impact of community participation especially within a relatively small community, and respecting clients' knowledge and understanding of their own world.

\section{Looking to the future}

Future care was perceived to be another significant issue for Korean parents raising children with disabilities in Aotearoa New Zealand - as it is for all families caring for a child with a disability. Korean parents with children with disabilities had significant concerns around their children's future care, and saw the need for culturally appropriate services. It was evident that Korean parents had a strong preference for having their 
children under their own care as long as possible, including when their children reach adulthood. This may be because traditional Koreans place more emphasis on interdependence among family members, and disability is perceived as a private family matter. Unlike New Zealanders who highlight independence and individualism, Koreans place greater emphasis on harmony with order and interdependence among family members and the community. In particular, supporting and caring for children, elderly, and sick family members are normal expectations for Korean people (Kim-Rupnow, 2005). Furthermore, services need to be able to reassure parents around their feelings of anxiety and mistrust.

Findings from the present study suggest that, in order to ensure culturally appropriate services in a multi-cultural society, there is a need for more culturally diverse professionals to be involved in the field. For instance, it is suggested that there is need to involve and employ more Korean professionals in mainstream health care fields in order to improve communication between carers and service users. A similar suggestion was raised in the study carried out by Hatton, Azmi, Caine, and Emerson (1998). They noted that, in order to improve services for Asian communities, there is a need to improve communication between Asian families and services. One of the suggestions they made to achieve such improvement, as recommended also by the participants in the present study, is to employ Asian staff with appropriate language skills in mainstream services to reduce difficulties around communication between carers and professionals. Though it cannot be argued that the same culture and ethnic background is necessary for every family, a good match with professionals of the same ethnicity is likely to result in positive outcomes and might be more effective.

Another suggestion was to establish an organisation for Koreans by Koreans.

Information from the data and discussions with participants suggest that creating and establishing an organisation for Korean children with disability staffed by Korean professionals and parents of those children may be beneficial. Participants in the present study expected that such services would reduce issues around culture, food and language, and provide more culturally appropriate services for the children and their families.

The limited evidence around the experiences of Korean immigrant parents raising children with disabilities in Aotearoa New Zealand suggests there are opportunities for further research in this area. Research around this topic may provide professionals and policy makers with research evidence that is specific to the local context which could guide practice with immigrant parents caring for children with disabilities. From the reflections in this present study, there are a number of ideas for further research on this topic. For instance, exploring the differing views of Korean parents and professionals around expectations of parents towards support services could be an area for further research. Also, as discussed, there is the tension between being disappointed with the services while also being pleased. However, it is not clear why such contrasts should exist; thus, this could be identified as an area for further work. Furthermore, since Korean services for Koreans are starting to emerge, such as group homes and after-care services, it may be valuable to explore the experiences, perceptions and expectations of parents currently using such services. It would also be valuable to conduct research on the ways in which Korean-provided services might be a positive development-on the development and provision of the services themselves. Such study might also reveal any potential negative sides of the initiative-for instance segregating Koreans families and their children further from mainstream society, and reducing choice for Korean families who remain excluded from the local Korean community. 


\section{Conclusion}

In conclusion, three important themes emerged, namely experience of services and its relationship with trust, cultural values and expectations, and looking to the future. The impacts of a lack of knowledge about the Aotearoa New Zealand health care system and available services, the language barrier, and the emotional challenges of trust in services provided among Korean parents with children with disabilities appeared frequently in the data. Contradictory perceptions around expectations on services between Korean parents and professionals were also found. The impacts of cultural values and expectations highlighted how Korean parents with children with disabilities view themselves as passive recipients of services. The last theme, looking to the future, explored parents' concerns around children's future care and the need for culturally appropriate services.

The present study recommends that professionals, service providers and government consider ways to build trusting relationships with Korean parents and their children with disabilities. In order to meet the needs of Korean families caring for a child with disabilities, greater attention needs to be placed on understanding expectations and having in place constructive dialogical processes that are culturally respectful. Further, ensuring better understanding around available services and around the general operation of the Aotearoa New Zealand health care system, training and educating volunteers, and increasing public awareness around disabilities are recommended. Professionals working with Korean parents with children with disabilities should seek opportunities to develop and increase parents' empowerment and self-advocacy. It is also suggested that employing more culturally diverse professionals in mainstream health care services, and advocating, empowering, and assisting culturally diverse professionals may be valuable. Further, active involvement of parents from minority communities in establishing culturally sensitive services for children with disabilities and their families is also encouraged as a way to achieve a fully inclusive society in multi-cultural Aotearoa New Zealand society. Yet, such initiatives must be approached with great care and consideration as the initiative may pose the potential risk of isolating minority communities further from the mainstream society, and limiting service provision options.

\section{References}

Bailey, D. B., Skinner, D., Rodriguez, P., Gut, D., \& Correa, V. (1999). Awareness, use, and satisfaction with services for Latino parents of young children with disabilities. Exceptional Children, 65(3), 367-389.

Bywaters, P., Ali, Z., Fazil, Q., \& Wallace, L. M. (2003). Attitudes towards disability amongst Pakistani and Bangladeshi parents of disabled children in the UK: Considerations for service providers and the disability movement. Health and Social Care in the Community, 11(6), 502-509. doi:10.1046/j.1365-2524.2003.00456.x

Cho, S., Singer, G., \& Brenner, M. (2000). Adaptation and accommodation to young children with disabilities: A comparison of Korean and Korean American parents. Topics in Early Childhood Special Education, 20, 236-249. doi: 10.1177/027112140002000404

Fatimilehin, I. A., \& Nadirshaw, Z. (1994). A cross-cultural study of parental attitudes and beliefs about learning disability (mental handicap). Mental Handicap Research, 7, 202-227. doi:10.1111/j.1468-3148.1994.tb00127.x

Hatton, C., Azmi, S., Caine, A., \& Emerson, E. (1998). Informal carers of adolescents and adults with learning difficulties from the South Asian communities: Family circumstances, service support and carer stress. British Journal of Social Work, 28, 821-837.

Holroyd, E. E. (2003). Chinese cultural influences on parental caregiving obligations toward children with disabilities. Qualitative Health Research, 13, 4-19.

Hsieh, H. F., \& Shannon, S. E. (2005). Three approaches to qualitative content analysis. Qualitative Health Research, 15(9), 1277-1288. doi:10.1177/1049732305276687

Hyun, K. J. (2001). Socio-cultural change and traditional values: Confucian values among Koreans and Korean Americans. International Journal of Intercultural Relations, 25(2), 203-229. doi:10.1016/S01471767(01)00009-8

Khanlou, N., Haque, N., Sheehan, S., \& Jones, G. (2015). "It is an issue of not knowing where to go": Service providers' perspectives on challenges in accessing social support and services by immigrant mothers of children with disabilities. Journal of Immigrant and Minority Health, 17(6), 1840-1847.

King, G., Desmarais, C., Lindsay, S., Piérart, G., \& Tétreault, S. (2015). The roles of effective communication and client engagement in delivering culturally sensitive care to immigrant parents of children with disabilities. Disability and Rehabilitation, 37(15), 1372-1381. 
Kim, B. S. K., Atkinson, D. R., \& Yang, P. H. (1999). The Asian Values Scale: Development, factor analysis, validation, and reliability. Journal of Counseling Psychology, 46, 342-352. Washington, D.C.: American Psychological Association.

Kim-Rupnow, W. (2005). Disability and Korean culture. In J. Stone (Eds.), Culture and disability: Providing culturally competent services (pp. 113-138). Thousand Oaks, CA: Sage Publications.

Lee, K. T. (1983). The structure of Korean consciousness, Korean series (1). [in Korean language] Seoul, Korea: Sinwon Moonwhasa.

Lindsay, S., Tétrault, S., Desmaris, C., King, G., \& Piérart, G. (2014). Social workers as "cultural brokers" in providing culturally sensitive care to immigrant families raising a child with a physical disability. Health \& Social Work, 39(2), 10-20.

Monette, D. R., Sullivan, T. J., \& DeJong, C. R. (2008). Applied social research: $A$ tool for the human services (7th ed.). Belmont, CA: Thomson/Wadsworth.

Park, I., \& Cho, L. (1995). Confucianism and the Korean family. [Special issue: Families in Asia: Beliefs and realities]. Journal of Comparative Family Studies, 26(1), 117-134.

Park, H., \& Chung, G. H. (2014). A multifaceted model of changes and adaptation among Korean mothers of children with disabilities. Journal of Child and Family Studies, 24(4), 915-929.

Shin, J. Y. (2002). Social support for families of children with mental retardation: Comparison between Korea and the United States. Mental Retardation, 40, 103-118.

Statistics New Zealand. (2006). New Zealand census of population and dwellings. Wellington, New Zealand: Author. Retrieved from http://m.stats.govt.nz/ Census/2013-census.aspx>

Vickers, M. H. (2006). Caring and working as work-home conflict. In M. H. Vickers (Ed.), Working and caring for a child with chronic illness: Disconnected and doing it all (pp. 15-33). New York, NY: Palgrave Macmillan.

Welterlin, A., \& LaRue, R. H. (2007). Serving the needs of immigrant families of children with autism. Disability \& Society, 22(7), 747-760. doi:10.1080/09687590701659600

Wong, S. Y., Wong, K. S., Martinson, I., Lai, A. C., Chen, W. J., \& He, Y. S. (2004). Needs of Chinese parents of children with developmental disability. Journal of Learning Disabilities, 8(2), 141-158. doi:10.1177/1469004704042703 